\title{
FITOSSOCIOLOGIA E DIVERSIDADE DE ESPÉCIES ARBÓREAS DAS PRAÇAS CENTRAIS DO MUNICÍPIO DE GURUPI-TO.
}

\author{
André Ferreira dos Santos ${ }^{1}$; Anderson Cleiton José2 Patrícia Aparecida de Sousa $^{3}{ }^{4}$.
}

\section{RESUMO}

As praças quando apresentam diversidade fitogeográfica e são bem distribuídas no interior das cidades formam um sistema de áreas verdes, que pelos seus atributos tendem promover o conforto térmico, acústico e visual. Sabendo desta necessidade o presente trabalho, teve como objetivo avaliar a composição florística e a diversidade de espécies arbóreas presentes nas praças centrais do município de Gurupi, localizada na região sul do estado do Tocantins, com $11^{\circ} 43^{\prime} 45^{\prime \prime}$ latitude sul e $49^{\circ} 04^{\prime} 07^{\prime \prime}$ longitude oeste. O estudo foi realizado durante o ano de 2009, entre os meses de março e dezembro. Avaliaram-se as espécies arbóreas localizadas nas praças centrais do município. Encontrou-se 261 árvores de 33 espécies e 15 famílias nas três praças estudadas, sendo 12 espécies nativas do bioma cerrado, com 24 árvores e 12 espécies exóticas à região estudada, num total de 237 árvores. Os autores observaram que as espécies com maior número de indivíduos são exóticas à região (Caesalpinia peltophoroides e Licania tomentosa); que a dominância de poucas espécies contribuiu para redução dos índices de diversidade; e que o uso de maior número de espécies, de preferência nativas da região em substituição às espécies exóticas é uma alternativa para o aumento dos índices de diversidade.

Palavras-chave: Árvores; Arborização urbana; Áreas verdes.

PHYTOSOCIOLOGY AND DIVERSITY OS ARBOREAL SPECIES IN SQUARES OF THE CENTRAL REGION OF GURUPI-TO.

\section{ABSTRACT}

The squares show when phytogeographic diversity and are well distributed within cities form a system of green areas, which by its attributes tend to promote thermal comfort, acoustic and visual. Knowing of this need the present work was to evaluate the floristic composition and diversity of tree species present in the central squares of the city of Gurupi, located in the southern state of Tocantins, with $11^{\circ} 43^{\prime} 45^{\prime \prime}$ south latitude and $49^{\circ} 04^{\prime} 07^{\prime \prime}$ longitude west. The study was conducted during 2009, between March and December. We evaluated tree species located in the central squares of the city: We found 261 trees of 33 species and 15 families in three sites studied 12 species native to the cerrado, with 24 trees and 12 species alien to the region studied, a total 237 trees. The authors observed that species with larger numbers of individuals are exotic to the region (Caesalpinia peltophoroides and Licania tomentosa), the dominance of few species contributed to lowering the levels of diversity, and that the use of most species, preferably native the region to replace the exotic species is an alternative to increasing the diversity indices.

Key-words: Three; Urban forestry; Green areas.

\footnotetext{
${ }^{1}$ Engenheiro Florestal, Professor Adjunto, Universidade Federal do Tocantins Campus Gurupi, Gurupi-TO. andrefs@uft.edu.br

${ }^{2}$ Engenheiro Florestal, Professor Adjunto, Universidade Federal de Lavras, Departamento de Ciências Florestais, Lavras-MG. acjose@dcf.ufla.br

${ }^{3}$ Engenheira Florestal, Professora Adjunta, Universidade Federal do Tocantins, Campus Gurupi, Gurupi-TO.patrícia@uft.edu.br

${ }^{4}$ recebido em 02.05.2013 e aceito para publicação em 15.12.2013
} 
O crescimento e desenvolvimento acelerado das cidades alteraram completamente a paisagem natural. Estas alterações principalmente de cunho antrópico, fez com que a vegetação fosse suprimida do ambiente urbano, cedendo lugar para edificações, pavimentação, entre outros equipamentos da malha urbana. Hoje, a vegetação urbana, que é considerada um elemento de vital importância não só para a composição da paisagem, como também desempenhando papel primordial na melhoria das condições ambientais, estão concentradas nas praças e distribuídas ao longo das ruas e das avenidas.

Para NUCCI (2001), a vegetação urbana deve cumprir três funções essenciais: a estética, ecológica e o lazer, as quais são indispensáveis para assegurar a qualidade ambiental das cidades, uma vez que as áreas verdes estabilizam as superfícies por meio da fixação do solo pelas raízes das plantas; reduz a velocidade do vento; protegem a qualidade da água, pois impedem que substâncias poluidoras escorram para os rios; filtram o ar, reduzindo os poluentes; diminuem a poeira em suspensão; e equilibram os índices de umidade no ar.

Para que a vegetação urbana propicie suas funções essenciais, é preciso que nesses redutos de áreas verdes as espécies arbóreas disponham de espaços adequados para que se desenvolvam e contemple certa diversidade entre as espécies plantadas.

Como exemplos destes redutos, temos as praças, as quais têm missão importante na vida da população urbana, promovendo o convívio social e o bem estar do cidadão.

Segundo PIVETTA et al. (2008) as localizações das praças são bastante variáveis; devendo ser bem distribuídas dentro do município, de maneira que cada bairro ou setor possua uma praça, ou seja, que cada área dessa, possa ser constituída de vegetação arbórea, a qual desempenhará funções ambientais importantes ao meio urbano.

Para DEMATTÊ (1997) as praças são espaços livres urbanos utilizados como local público, as quais além de incentivar a socialização e oferecer opções de lazer, é a forma de que a população tem de estar em contato direto com a natureza.

As praças uma vez apresentando uma diversidade fitogeográfica e sendo bem distribuídas no interior das cidades vão formar um sistema de áreas verdes, que pelos seus atributos tendem a promover o conforto térmico, acústico e visual (RESENDE et al., 2009).

De acordo com MILANO (1988) a ausência de diversidade de espécies arbóreas na área urbana pode acarretar diversos problemas, como a fixação da fauna, a manutenção do equilíbrio biológico e controle de pragas.

Já para GUZZO (1999) a ocorrência da diversidade fitogeográfica no ambiente urbano promove maior estabilidade ecológica, onde os novos habtitats propiciam o surgimento de outras espécies (flora e fauna) no processo de desenvolvimento e interações ecológicas, caracterizadas pela competição, predação e simbiose, o que dificulta o aparecimento de pragas que coloquem em riscos a vida das populações.

KAGEYAMA (2009) diz que a escolha criteriosa das espécies e a diversidade delas é uma preocupação recente, já que a principal preocupação na escolha era até então muito mais para a beleza das folhagens ou de suas flores, do que a tentativa de recuperação de algo da natureza. Assim, é também muito comum que muito poucas espécies sejam predominantes na arborização de uma cidade, 
inclusive com muito baixa variabilidade genética para as mesmas, provavelmente com a ocorrência de endogamia e problemas genéticos nessas populações.

Dessa forma, a escolha e utilização de espécies com maior diversidade poderia ser referência para o uso do recurso vegetal nas cidades, de modo a trazer não só beleza e conforto térmico aos que ali vivem, mas também um equilíbrio ecológico e aumento da biodiversidade nesses ambientes tão duros e inóspitos (KAGEYAMA, 2009).

Sabendo da necessidade de uma cidade apresentar áreas verdes com uma vegetação arbórea diversificada e de qualidade, oferecendo assim qualidade de vida a sua população o presente trabalho, teve como objetivo avaliar a composição florística e a diversidade de espécies arbóreas presentes nas praças centrais do município de Gurupi-TO.

\section{MATERIAL E MÉTODOS}

\section{Localização e caracterização da área do estudo}

A cidade de Gurupi com uma população estimada em 74.357 habitantes (IBGE, 2009) está localizada na região sul do estado do Tocantins, à aproximadamente 287 metros de altitude, $11^{\circ} 43^{\prime} 45^{\prime \prime}$ latitude sul e $49^{\circ} 04^{\prime} 07^{\prime \prime}$ longitude oeste. O clima segundo a classificação de Koppen é do tipo Aw (tropical, com temperatura média do mês mais frio do ano maior de $18^{\circ} \mathrm{C}$, com chuvas de verão e estiagem de inverno). As médias históricas locais mostram temperatura média máxima de $32,6{ }^{\circ} \mathrm{C}$ e média mínima de $20,7{ }^{\circ} \mathrm{C}$ e precipitação média anual de $1735,5 \mathrm{~mm}$.

A região está localizada no domínio das Savanas (Cerrado), caracterizada pela predominância de vegetação xeromorfa aberta, dominada e marcada por um estrato herbáceo (IBGE, 1992; MIRANDA e BOGNOLA, 1998). A cidade é um pólo de desenvolvimento agrícola e de pecuária de corte o que contribuiu para a supressão de grande parte da vegetação original.

O estudo foi realizado durante o ano de 2009, entre os meses de março e dezembro. Foram estudadas as espécies arbóreas localizadas nas praças centrais do município: Praça João Borges Leitão (Igreja Abadia), Praça da Igreja Santo Antônio e Praça Francisco Henrique de Santana. (Figura 1). Todas as espécies com indivíduos apresentando mais de $15 \mathrm{~cm}$ de diâmetro e $5 \mathrm{~m}$ de altura foram contados e identificados através de consulta a bibliografia, herbários e especialistas. A classificação das famílias botânicas segue a Classificação APG (APG III, 2009).

A determinação das dimensões das praças foi feita com o programa SPRING (CÂMARA et al., 1996), fazendo-se a remoção da área de edificações para o cálculo da área total de cada praça.

A classificação das espécies quanto à origem baseou-se na descrição das espécies quanto ao seu habitat natural. Foram feitas consultas em bibliografias especializadas (CORREIA, 1984; LORENZI, 2002; CARVALHO, 2003; LORENZI et. a., 2003; CARVALHO 2006; CARVALHO 2008) e consulta a herbários. Espécies que não possuíam espécimes coletados ou descritos por bibliografia em áreas de Savana foram consideradas

André Ferreira dos Santos et al. 
Figura 1. Localização das praças centrais do município de Gurupi-TO, (1- Praça João Borges Leitão, 2- Praça da Igreja Santo Antônio e 3 Praça Francisco Henrique de Santana
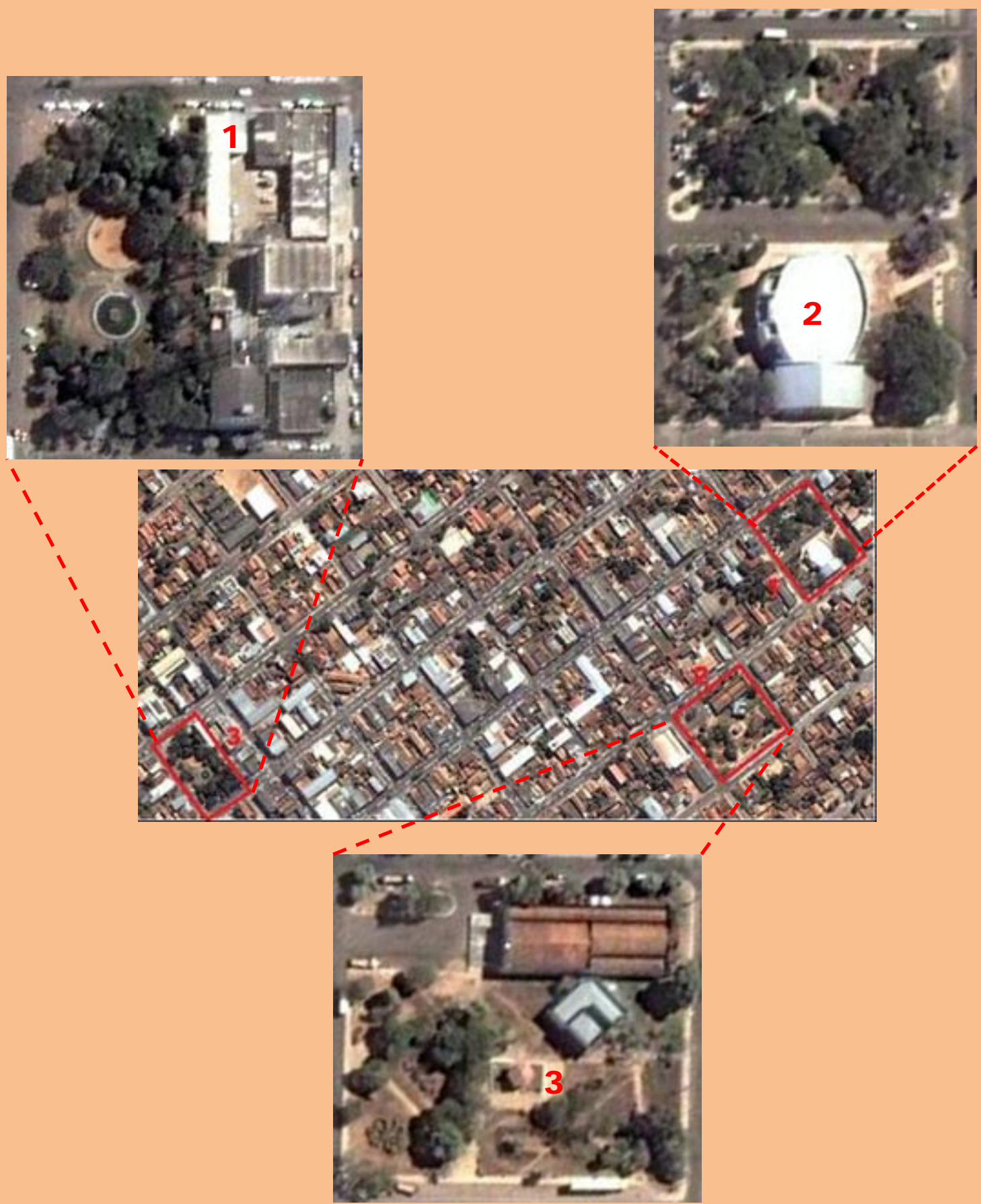

Fonte: Google Earth / Source: Google Earth. FITOSSOCIOLOGIA E DIVERSIDADE DE ESPÉCIES ARBÓREAS DAS... 
Foram calculados para cada espécie a densidade absoluta (DA), densidade relativa (DR), frequência absoluta (FA) e frequência relativa (FR), onde:

$$
\mathrm{DA}=\frac{n}{h a}
$$

n: número de indivíduos amostrados de cada espécie;

ha: hectare.

$$
\mathrm{DR}=\frac{n / h a}{N / h a} \times 100
$$

n: número de indivíduos amostrados de cada espécie;

$\mathrm{N}$ : Número total de indivíduos amostrados, de todas as espécies do levantamento; ha: hectare.

$\mathrm{FA}=\frac{\text { Número de praças com ocorrência da espécie }}{\text { Número total de praças }}$

$$
\mathrm{FR}=\frac{F A i}{\sum F A} \times 100
$$

FAi: Frequência absoluta da i-ésima espécie;

$\sum F A$ : Somatório da frequência absoluta de todas as espécies.

A diversidade global de espécies foi medida através do índice de diversidade de Shannon-Weaver (H') e índice de dominância de Simpson (C), utilizando o programa PAST (HAMMER et. al., 2001), onde:

$$
\mathrm{H}^{\prime}=\frac{\left[N \cdot \ln (N)-\sum_{i=1}^{S} n_{i} \cdot \ln \left(n_{i}\right)\right]}{N}
$$

H': Índice de diversidade de Shannon-Weaver;

i: $1 \ldots \mathrm{N}$

S: Número de espécies amostradas;

$\mathrm{n}_{\mathrm{i}}$ : Número de indivíduos amostrados da i-ésima espécie;

$\mathrm{N}$ : Número total de indivíduos amostrados;

$\ln$ : Logarítmo neperiano.

$\mathrm{C}=\frac{\sum_{i=1}^{S} n_{i}\left(n_{i}-1\right)}{N(N-1)}$

$\mathrm{C}=$ Índice de dominância de Simpson;

$\mathrm{n}_{\mathrm{i}}=$ Número de indivíduos amostrados da i-ésima espécie

$\mathrm{N}=$ Número total de indivíduos amostrados

$\mathrm{S}=$ Número total de espécies amostradas 
Quantidade de espécies nas praças

Foram encontradas 261 árvores de 33 espécies e 15 famílias nas três praças estudadas, sendo 12 espécies nativas do bioma cerrado, com 24 árvores e 12 espécies exóticas à região estudada, num total de 237 árvores (Tabela 1).

Tabela 1. Famílias, espécies, número total de indivíduos (NTI), densidade absoluta (DA), densidade relativa (DR), freqüência absoluta (FA), freqüência relativa (FR) e origem de espécies arbóreas encontradas nas três praças centrais do município de Gurupi-TO. Origem: $\mathrm{E}=$ exótica, $\mathrm{N}=$ nativa.

\begin{tabular}{|c|c|c|c|c|c|c|c|}
\hline Família/Espécie & $\begin{array}{c}\text { Nome } \\
\text { popular }\end{array}$ & NTI & DA & $\begin{array}{l}\text { DR } \\
(\%)\end{array}$ & FA & FR (\%) & Origem \\
\hline \multicolumn{8}{|l|}{ Anacardiaceae } \\
\hline Anacardium occidentale L. & Cajueiro & 3 & 1,36 & 1,15 & 0,67 & 4,17 & $\mathrm{~N}$ \\
\hline Mangifera indica $L$ & Mangueira & 6 & 2,73 & 2,30 & 0,67 & 4,17 & $\mathrm{E}$ \\
\hline \multicolumn{8}{|l|}{ Apocynaceae } \\
\hline Plumeria rubra & $\begin{array}{c}\text { Jasmim- } \\
\text { manga }\end{array}$ & 1 & 0,45 & 0,38 & 0,33 & 2,08 & $\mathrm{E}$ \\
\hline \multicolumn{8}{|l|}{ Arecaceae } \\
\hline Caryota urens & $\begin{array}{l}\text { Rabo-de- } \\
\text { peixe }\end{array}$ & 2 & 0,91 & 0,77 & 0,33 & 2,08 & $\mathrm{E}$ \\
\hline Livistona chinensis & $\begin{array}{c}\text { Palmeira-de- } \\
\text { leque }\end{array}$ & 2 & 0,91 & 0,77 & 0,33 & 2,08 & $\mathrm{E}$ \\
\hline Phoenix roebelenii & Fênix & 4 & 1,82 & 1,53 & 0,33 & 2,08 & $\mathrm{E}$ \\
\hline Roystonea oleracea & $\begin{array}{l}\text { Palmeira- } \\
\text { real }\end{array}$ & 16 & 7,28 & 6,13 & 0,67 & 4,17 & $\mathrm{E}$ \\
\hline Syagrus oleracea & Gueroba & 6 & 2,73 & 2,30 & 0,67 & 4,17 & $\mathrm{~N}$ \\
\hline \multicolumn{8}{|l|}{ Bignoniaceae } \\
\hline Tabebuia impetiginosa & Ipê-roxo & 2 & 0,91 & 0,77 & 0,67 & 4,17 & $\mathrm{~N}$ \\
\hline Tabebuia roseo-alba & Ipê-branco & 1 & 0,45 & 0,38 & 0,33 & 2,08 & $\mathrm{~N}$ \\
\hline Tabebuia serratifolia & Ipê-amarelo & 3 & 1,36 & 1,15 & 0,33 & 2,08 & $\mathrm{~N}$ \\
\hline Tecoma stans & Ipê-mirim & 6 & 2,73 & 2,30 & 0,67 & 4,17 & $\mathrm{E}$ \\
\hline \multicolumn{8}{|l|}{ Caricaceae } \\
\hline Carica papaya & Mamoeiro & 1 & 0,45 & 0,38 & 0,33 & 2,08 & $\mathrm{E}$ \\
\hline \multicolumn{8}{|l|}{ Casuarinaceae } \\
\hline Casuarina equisetifolia $J, R, \&$ & Casuarina & 2 & 0,91 & 0,77 & 0,33 & 2,08 & $\mathrm{E}$ \\
\hline
\end{tabular}


Forst

Chrysobalanaceae

Licania tomentosa

Oiti

22,74

19,16

1,00

6,25

E

Combretaceae

Terminalia catappa L,

Sete-copas

13

5,91

4,98

0,67

4,17

E

Fabaceae

Anadenanthera colubrina (Vell.)

Brenan

Bauhinia forficata Link

Caesalpinia peltophoroides

Benth.

\section{Angico-}

vermelho

$1 \quad 0,45$

0,38

0,33

2,08

Pata-de-vaca

$9 \quad 4,09$

$3,45 \quad 0,67$

4,17

E

Sibipiruna

98

44,57

37,55

1,00

6,25

Cássia-

E

Cassia fistula $L$.

chuva-de-

0,45

0,38

0,33

2,08

Copaifera langsdorfii

Enterolobium contortisiliquum

Pterodon polygaeflorus

$$
\text { ouro }
$$

Pau-d'óleo

0,45

0,38

0,33

2,08

Tamboril

0,45

0,38

0,33

2,08

Sucupira-

branca

$0,77 \quad 0,33 \quad 2,08$

$\mathrm{N}$

$\mathrm{N}$

$\mathrm{N}$

Malvaceae

Chorisia speciosa A. St.-Hil.

Hibiscus rosa-sinensis $L$,

Pachira aquatica

Sterculia striata

Paineira
Graxa-de-
estudante
Munguba
Chicá

$2 \quad 0,91$

$$
2,08
$$

Meliaceae

Azadirachta indica

Nim-indiano

$1 \quad 0,45$

$0,38 \quad 0,33 \quad 2,08$

E

$5 \quad 2,27$

1,92

0,33

2,08

E

$\mathrm{N}$

$0,38 \quad 0,33 \quad 2,08$

$0,38 \quad 0,33 \quad 2,08$

$\mathrm{N}$

Moraceae

Ficus benjamina

Myrtaceae

Psidium guajava L,

Syzygium malaccense

$$
\text { Figueira }
$$

$\begin{array}{ll}1 & 0,45 \\ 1 & 0,45\end{array}$

0,38

2,68

0,33

2,08

E

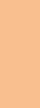

$\begin{array}{ccccccc}\text { Goiabeira } & 3 & 1,36 & 1,15 & 0,67 & 4,17 & \text { E } \\ \begin{array}{c}\text { Jambo- } \\ \text { vermelho }\end{array} & 1 & 0,45 & 0,38 & 0,33 & 2,08 & \text { E }\end{array}$

\section{Nyctaginaceae}

Bougainvillea glabra

Três-marias

$6 \quad 2,73$

$2,30 \quad 0,67$

4,17

E

Rubiaceae

Genipa Americana

Genipapo

$2 \quad 0,9$

$0,77 \quad 0,67$

4,17

N

TOTAL

261

100

16

100


As espécies com maior representatividade na área, expressa pela densidade relativa, foram: Caesalpinia peltophoroides (37,55\%) e Licania tomentosa $(19,16 \%)$, as quais juntas representam $56,71 \%$ de todos os indivíduos encontrados. Essas espécies foram encontradas em todas as praças estudadas $(\mathrm{FA}=1)$.

Um fato que merece ser destacado sobre a arborização nesse estudo é o alto índice de espécies exóticas, totalizando $90,8 \%$ dos indivíduos, o que evidência uma escolha por espécies para atender um propósito em particular, que no caso da cidade de Gurupi, pode ser observado para a sibipiruna e o oiti.

A sibipiruna (Caesalpinia peltophoroides) é nativa da mata atlântica, semidecídua, de rápido crescimento e florescimento ornamental. De excelente efeito paisagístico, a sibipiruna fornece uma sombra fresca e floração exuberante. Apesar do porte grande e desenvolvimento rápido, ela é comportada e não produz raízes agressivas, desta forma é boa opção para arborização urbana, na ornamentação de vias públicas, praças e até mesmo em calçamentos (PATRO, 2012). A cidade de Maringá, no Paraná, possui arborização com 80\% em sibipiruna (WIKIPEDIA, 2012).

O oiti (Licania tomentosa) é originária da Região Nordeste do Brasil, é muito utilizada na arborização de várias cidades brasileiras, como o Rio de Janeiro, por exemplo, (WIKIPEDIA, 2012). A espécie forma uma bela copa frondosa e as raízes não são agressivas. Por isso ela é indicada para arborização de jardins, praças, avenidas e ruas. Muito rústico, o Oiti resiste bem à poluição e por isso sobressai entre as espécies usadas na arborização urbana. Muito utilizada na arborização urbana de cidades do norte do país e de regiões litorâneas (BRAGA, 2010). Gurupi por ser uma cidade de clima tropical, acredita-se que também tenha sido plantada para o fornecimento de sombra, pois essa é uma espécie que mantém as folhas durante todo o ano, inclusive no período seco.

A utilização de espécies exóticas na arborização de praças, avenidas, bairros, é comum nas cidades brasileiras. O que foi confirmado por SILVA et al. (2007) em inventário da arborização dos bairros Bancários, Pinheiros e Brasília, de Pato Branco, no Paraná, onde apresentou uma frequência de $81,3 \%$ de indivíduos arbóreos exóticos. SILVA et al. (2007) encontrou $62,4 \%$ de espécies exóticas no bairro Centro de Pato Branco, Paraná. LINDERNMAIER \& SANTOS (2008) encontraram $58 \%$ de espécies exóticas, em Cachoeira do Sul, no Rio Grande do Sul, na avaliação das praças desta cidade.

Para LINDERNMAIER \& SANTOS (2008) a super utilização de espécies exóticas na arborização de áreas verdes urbanas, pode ser atribuída em parte a um reflexo de tendências paisagísticas anteriores, pois, sob o ponto de vista estético, simplesmente é mais fácil encontrar espécies de grande beleza distribuídas por todo o mundo, do que somente em um espaço geográfico ou formação vegetal restrita. Também há um evidente desconhecimento por parte da população e órgãos governamentais acerca da riqueza e utilização de espécies de nossa flora.

As famílias botânicas que apresentaram destaque em riqueza de espécies foram Fabaceae e Arecaceae, com 7 e 5 espécies, respectivamente (Tabela 1). De acordo com LINDERNMAIER \& SANTOS (2008) a família Fabaceae apresentou maior riqueza de espécies em Cachoeira do Sul, RS. As famílias Apocynaceae, Caricaceae, Casuarinaceae, Chrysobalanaceae, Combretaceae, Meliaceae, Moraceae, Nyctaginaceae e Rubiaceae, apresentaram apenas 1 espécie. Embora a família Chrysobalanaceae tenha contribuído com um total de 50 indivíduos. 


\section{Distribuição das espécies nas praças e Diversidade de espécies}

A praça que apresentou a maior quantidade de espécies foi a Praça da Feira da Amizade (113 indivíduos), seguida pela Praça Santo Antônio (85 indivíduos) e Praça da Abadia (63 indivíduos).

Em todas as Praças as espécies dominantes foram C. peltophoroides e L. tomentosa, exceto na Praça da Abadia onde C. peltophoroides (25 indivíduos) e Roystonea oleracea (14 indivíduos), foram às espécies com maior número de indivíduos.

A maioria dos estudos realizados em áreas urbanas aborda somente a florística, e em alguns casos a fitossociologia. Baseado em estudos fitossociológicos alguns autores recomendam um valor máximo de 10 a $15 \%$ indivíduos da mesma espécie, principalmente por questões sanitárias (GREY \& DENEKE, 1978). Estudos sobre diversidade de espécies em áreas urbanas ainda são escassos, o que dificulta fazer qualquer tipo de comparações.

A diversidade de espécies medida pelo índice de Shannon-Wiener (H') foi de 2,37 e a dominância medida pelo índice de Simpson (C) foi de 0,81 . Apesar da dificuldade de comparações para esses valores, percebe-se a importância da substituição de espécies dominantes, as quais ocorreram em todas as praças por espécies nativas como forma de aumentar a diversidade local e reduzir a dominância de espécies.

Em trabalho semelhante avaliando a diversidade de espécies em praças do município de Cachoeira do Sul-RS, LINDENMAIER \& SANTOS (2008) encontraram o valor de diversidade 3,86 (H') e sugeriram o uso do índice de diversidade para avaliações futuras na mesma área estudada.

\section{CONCLUSÕES}

As espécies com maior número de indivíduos na área estudada são exóticas à região (Caesalpinia peltophoroides e Licania tomentosa); a dominância de poucas espécies contribuiu para redução dos índices de diversidade nas praças estudadas; e o uso de maior número de espécies, de preferência nativas da região em substituição às espécies exóticas é uma alternativa para o aumento dos índices de diversidade.

\section{REFERÊNCIAS BIBLIOGRÁFICAS}

APG III. An update of the Angiosperm Phylogeny Group classification for the orders and families of flowering plants: APG III. Botanical Journal of the Linnean Society. v.161 (2): 105-121. 2009.

BRAGA, R.C. Oiti (Licania Tomentosa). Bromélias e Plantas Exóticas. 2010. Disponível em: $<$ http://pro.casa.abril.com.br/group/produtoresecolecionadoresdebromliaseorqudeas/forum/topics/oiti-licaniatomentosa?xg source=activity>. Acessado em: 2 junho 2012. 
CÂMARA, G.; SOUZA, R.C.M.; FREITAS, U.M.; GARRIDO, J.C.P. SPRING: Integrating Remote Sensing and GIS with Object-Oriented Data Modelling. Computers and Graphics, v.15, p.13-22, 1996.

CARVALHO, P.E.R. Espécies arbóreas brasileiras. Brasília: Embrapa Informação Tecnológica, 2003, v. 1, 1039 p.

CARVALHO, P.E.R. Espécies arbóreas brasileiras. Ed. Brasília: Embrapa Informação Tecnológica, 2006, v. $2,627 \mathrm{p}$.

CARVALHO, P.E.R. Espécies arbóreas brasileiras. Ed. Brasília: Embrapa Informação Tecnológica, 2008 , v. $3,593 p$.

CORREA, M.P. Dicionário das plantas úteis do Brasil e das exóticas cultivadas. (6v.) Rio de Janeiro: IBDF, 1984.

DEMATTÊ, M.E.S.P. Princípios de Paisagismo, Série Paisagismo 1, Unesp, Funep. Jaboticabal, 1997, 104 p.

GREY, G.W. e DENEKE, F. Urban forestry. New York: John Wiley, 1978.

GUZZO, P. Estudo dos espaços livres de uso público da cidade de Ribeirão Preto/SP, com detalhamento da cobertura vegetal e áreas verdes de dois setores urbanos. Rio Claro - SP. 1999. 125p. Dissertação (Mestrado em Geociências e Meio Ambiente), Universidade Estadual Paulista, Rio Claro, 1999.

HAMMER, Ø.; HARPER, D.A.T.; RYAN, P.D. PAST: Paleontological Statistics Software Package for Education and Data Analysis. Paleontologia Eletrônica 4(1): 9p. 2001.

IBGE. IBGE Cidades - Estimativa da população 2009. Disponível em: http://www.ibge.gov.br/cidadesat/topwindow.htm?1. Acessado em 03/04/2010.

IBGE. Manual técnico da vegetação brasileira - Séries Manuais técnicos em geociências, n. 1. Rio de Janeiro: IBGE, 1992. 92p.

KAGEYAMA, P. Arborização urbana, biodiversidade e diversidade genética de espécies. In: Congresso Brasileiro de Arborização Urbana, XIII, 2009, Rio Branco. Anais eletrônicos... Acre, 2009. Disponível $\mathrm{m}:<\mathrm{http}: /$ www.cbau2009.com.br/RESUMO.../Artigo\%20Paulo\%20Kageyama.pdf Acessado em 31 de mar. 2010.

FITOSSOCIOLOGIA E DIVERSIDADE DE ESPÉCIES ARBÓREAS DAS... 
LINDERNMAIER, D.S \& DOS SANTOS, N. O. Arborização Urbana das praças de Cachoeira do Sul-RSBrasil: Fitogeografia, Diversidade e Índice de áreas verdes. Pesquisas, Botânica.

São Leopoldo, n. 59, p. 307-320, 2008.

LORENZI, H. Árvores Brasileiras: manual de identificação e cultivo de plantas arbóreas nativas do Brasil. 2 ed. Nova Odessa: Instituto Plantarum, 2002, v. 2, 382p.

LORENZI, H. Árvores Exóticas do Brasil: madeireiras, ornamentais e aromáticas, 1 ed. Nova Nova Odessa: Instituto Plantarum, 2003, 384p.

MILANO, M.S. Avaliação quali-quantitativa e manejo da arborização urbana: o exemplo de Maringá/ PR. Curitiba, 1988, 120 p. Tese (Doutorado em Engenharia Florestal) Setor de Ciências Agrárias, UFPR, Curitiba, 1988.

MIRANDA, E.E. de; BOGNOLA, I.A. Nota sobre o andamento do Zoneamento Agroecológico do Estado do Tocantins: Parecer técnico. Campinas: Embrapa-CNPM, 1998. 3p. Embrapa Monitoramento por Satélite-AMT.

NUCCI, J. C. Qualidade Ambiental e Adensamento Urbano: Um Estudo de Ecologia e Planejamento da Paisagem Aplicada ao Distrito de Santa Cecília. São Paulo: Ed. Humanitas. 2001. 236p.

PATRO, R. Sibipiruna - Caesalpinia peltophoroides. Disponível em:< http://www.jardineiro.net/br/banco/caesalpinia_peltophoroides.php>. Acesso em: 02 julho 2012.

PIVETTA, K.F.L.; PAIVA, P.D.O.; NERI, F.C.S. Paisagismo em grandes espaços. In: PAIVA, P.D.O. Paisagismo; conceitos e aplicações. Lavras: UFLA, 2008, p.179-211.

RESENDE, W. X.; et al. Índice de Áreas Verdes Públicas: uma avaliação fitogeográfica da qualidade ambiental em Aracaju. In: Simpósio Brasileiro de Geografia Física Aplicada, XIII, 2009, Viçosa. Anais eletrônicos... Viçosa, 2009. Disponível em:

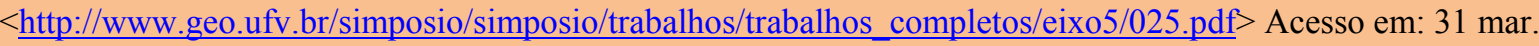
2010.

SILVA, L.M.; et al. Arborização de vias públicas e a utilização de espécies exóticas: o caso do Bairro Centro de Pato Branco/PR. Scientia Agraria, Curitiba, v.8, n.1, p.47-53. 2007.

WIKIPEDIA. Oiti. Disponível em: <http://pt.wikipedia.org/wiki/Oiti>. Acesso em: 02 julho 2012.

WIKIPEDIA. Sibipiruna. Disponível em: < http://pt.wikipedia.org/wiki/Sibipiruna $>$. Acesso em: 02 julho 2012. 